\title{
UK Renal Registry 16th Annual Report: Chapter 6 Demographics and Outcomes of Patients from Different Ethnic Groups on Renal Replacement Therapy in the UK
}

\author{
Udaya Udayaraj $^{\mathrm{a}}$, Rishi Pruthi ${ }^{\mathrm{b}}$, Anna Casula ${ }^{\mathrm{b}}$, Paul Roderick ${ }^{\mathrm{c}}$

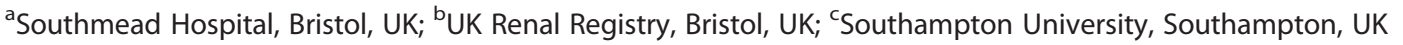

\section{Key Words}

Access - Demography - Ethnic group - Ethnicity - Hospitalisation - Incidence $\cdot$ Outcome $\cdot$ Survival · Transplantation

\begin{abstract}
Introduction: This chapter describes the patient characteristics and outcomes for the three main ethnic groups (White, South Asian, Black) on renal replacement therapy (RRT) in the UK. Methods: Data on patients ( $>18$ years old) from all 71 UK adult renal centres starting RRT between 2003 and 2012 were considered. Scottish centres were excluded due to poor ethnicity data. Results: The age-gender standardized incidence ratio of RRT was higher (2-3 times) in regions with a high ethnic minority population compared to those with a low ethnic minority population. South Asian and Black patients were significantly younger than Whites; had more diabetes causing established renal failure and lived in more deprived areas. The proportion of patients with at least one comorbidity was greater amongst White patients compared to South Asian and Black patients.
\end{abstract}

The proportion of patients starting PD and having preemptive transplantation was lower amongst both ethnic minorities. The attainment of various laboratory standards was comparable or better for the ethnic minorities compared to White patients except for calcium standard attainment (for South Asians) and haemodialysis dose attainment (for Black patients). Compared to White patients, both ethnic minorities had similar rates of listing for deceased donor kidney transplantation but had lower rates of transplantation once wait-listed, and lower rates of living kidney donor transplantation. One and five year kidney allograft adjusted survival was poorer for Black patients but similar for South Asians compared to White patients. Black and South Asian patients had a better adjusted survival on dialysis compared to White patients. Conclusions: The persistent high incidence of RRT, the better survival on dialysis and the poor access to kidney transplantation for South Asian and Black patients and early allograft loss for Black patients will impose a disproportionate demand on dialysis provision in those areas with a high ethnic minority population.

$\begin{array}{ll}\text { KARGER } & \text { C 2014 S. Karger AG, Basel } \\ \text { Fax +4161306 1234 } & \text { 1160-2110/14/1254-0111\$38.00/0 } \\ \begin{array}{l}\text { E-Mail karger@karger.com } \\ \text { www.karger.com }\end{array} & \begin{array}{l}\text { Accessible online at: } \\ \text { www.karger.com/nec }\end{array}\end{array}$

Udaya Udayaraj

UK Renal Registry, Southmead Hospital, Southmead Road, Bristol, BS10 5NB, UK

Email: renalregistry@renalregistry.nhs.uk 


\section{Introduction}

The ethnic minority population in the UK has increased from $9.7 \%$ in the 2001 Census to $13 \%$ in the most recent 2011 Census [1]. Although the ethnic make-up of the UK is increasingly diverse, this chapter mainly reports on the characteristics and comparisons of patient level outcomes of those on renal replacement therapy (RRT) from the three main ethnic groups: White, South Asian and Black. Patients from other ethnic groups were a heterogeneous population and accounted for a small proportion of all patients on RRT and therefore are not discussed in detail in this chapter.

\section{Methods}

Data on patients ( $>18$ years old) from all 71 UK adult renal centres starting RRT between 2003 and 2012 and who did not recover renal function within 90 days and who had data on ethnicity were considered. Centres in Scotland were excluded from further analysis due to poor ethnicity data completeness (15.3\%). The patient cohort used for the various analyses differ slightly, and these variations are described in the individual sections. Details of ethnicity coding used by centres and regrouping of these codes by the UK Renal Registry (UKRR) can be found in appendix $\mathrm{H}$ at www.renalreg.com.

Regional variations in RRT incidence rates by ethnic group

Data completeness for ethnicity for patients on RRT has improved over the years. The proportion of patients with missing ethnicity data has decreased from $13.3 \%$ in 2003 to 3\% in 2012. As missing ethnicity data would bias the estimates of incidence rates in a population, only patients starting RRT in the years 2010-2012 ( $\sim 98 \%$ ethnicity data completeness) were included in this analysis. Details of methods used to calculate age-gender standardized incidence rates can be found in appendix D at www.renalreg.com. As census data for Northern Ireland population by ethnic group was not available for all age groups above 65 years, Northern Ireland centres were excluded from analyses that required age-gender standardization.

\section{Demographics and clinical characteristics}

All patients starting RRT between 2003 and 2012 with data on ethnicity from centres in England, Wales and Northern Ireland were included for these analyses. The following patient characteristics at start of RRT were studied: age, gender, social deprivation, primary renal diagnosis, comorbidity, estimated glomerular filtration rate (eGFR), haemoglobin ( $\mathrm{Hb})$, time between first seen at renal centre and start of RRT $(<90$ days, 90-365 days, $>365$ days), and treatment modality at start, 90 days and at 1 year from start of RRT.

Details of EDTA coding for primary renal diagnosis used by renal centres can be found in appendix $\mathrm{H}$ at www.renalreg.com. Details of comorbid conditions listed in the UKRR dataset and their regrouping are described elsewhere [2]. Social deprivation was measured at super output area level using the adjusted Index of Multiple Deprivation (IMD) [3]. The super output areas were sorted by their IMD score and divided into quintiles, a high IMD quintile indicating a higher level of deprivation. Each patient was allocated an IMD score and a quintile by matching their postcode of residence to the 2001 Census lower layer super output area.

eGFR was calculated using the 4 variable MDRD study equation [4] using the most recent creatinine data that was available within 14 days before start of RRT. Similarly, the most recent $\mathrm{Hb}$ data within 14 days before start of RRT only were included in the analyses.

Chi-squared and Kruskal Wallis tests were used to compare groups where appropriate.

\section{Patient outcome measures}

1) Attainment of laboratory standards on dialysis

Only patients who started RRT from 2003 to 2011 and were on dialysis at the end of their first year of RRT were included in the analyses. Values from the 4 th quarter (or 3 rd quarter if 4 th quarter reading not available) in the first year of RRT for each of the following variables were used to ascertain achievement of standards set by the UK Renal Association: Hb 100-120 g/L; phosphate (PO4) 1.1-1.7 mmol/L; corrected calcium (Ca) 2.2-2.5 mmol/L; parathyroid hormone $(\mathrm{PTH}) 16-72 \mathrm{pmol} / \mathrm{L}$; urea reduction ratio (URR) $>65 \%$. Patients who did not have a recorded value for a laboratory variable either in the $3 \mathrm{rd}$ or 4 th quarter in their first year of RRT were excluded from the analysis for that laboratory standard. For patients on HD, all the variables were measured pre-dialysis. For the analysis on URR in HD patients, patients on home dialysis and those who received less than three dialysis sessions per week were not included. Logistic regression analyses were performed to compare attainment of standards between ethnic groups adjusting for age $(<35,35-44,45-54,55-64,65-$ $74,75+$ years), gender, primary renal diagnosis, year of start of RRT, dialysis modality at one year, IMD quintile and centre as fixed effect. Adjustments for comorbidity were not performed due to incomplete data.

\section{2) Access to kidney transplantation}

The UKRR has previously reported on access to transplantation for the various ethnic groups in the UK and the detailed methodology is described in those reports $[5,6,7]$.

\section{3) Kidney transplant outcomes}

Kidney allograft survival and allograft function amongst those with a functioning graft at one year and five years were compared between the ethnic groups. For those who had more than one kidney transplant during the study period, only the first transplant episode was included in the analyses. For the one year graft outcomes analyses, patients who had a kidney only transplant and who had data on ethnicity, IMD score, primary renal diagnosis and donor type between 2003 and 2011 were included. For the five year graft outcome analyses, patients who had a kidney only transplant between 2003 and 2007 were included in the analyses to allow five year follow up for all patients. Kaplan-Meier analyses with and without censoring for death were performed to compare unadjusted graft survival between ethnic groups. Cox proportional hazards model censoring for death, and death with functioning 
graft as a competing event were performed adjusting for age at transplant as a continuous variable, gender, primary renal diagnosis, IMD quintile, year of transplant, time on RRT prior to transplantation and type of donor (post brain stem death donor versus post circulatory death donor versus live donor). Other donor details such as donor age, cold ischaemia time, human leucocyte antigen (HLA) mismatch were not available to be included in the adjusted analyses.

Graft function amongst those with a functioning graft at one year and five years was estimated using the CKD-EPI equation [8] from the most recent serum creatinine available in the last quarter of the first and fifth years post kidney transplantation respectively.

4) Patient survival on dialysis

Patients who started RRT between 2003 and 2012 (excluding patients in the last quarter of 2012 to allow at least 90 days of RRT) and who had data on ethnicity were considered. Unadjusted survival at 90 days from start of RRT, one year from start of RRT and one year after 90 days from start of RRT is reported. For the one year after 90 day survival analyses, patients who started RRT from the last quarter of 2011 were not included to allow adequate follow up. Kaplan Meier analyses and a Cox proportional hazards model adjusting for age as a continuous variable, gender, centre as random effect, year of RRT start and IMD quintile were used with and without censoring for transplantation to compare survival after 90 days from RRT start between the ethnic groups. Due to non-proportionality, stratified analyses were performed by primary renal disease (diabetic, non-diabetics), age group $(<45$, $45-64,65$ years) and dialysis modality at day 90 from RRT start. Patients were followed up until 31st December 2012 or death if earlier.

The EDTA codes for causes of death were used by centres and these can be found in appendix $\mathrm{H}$ at www.renalreg.com

There was no significant difference between those who were included and excluded due to missing ethnicity data except that the cohort without an ethnic code was older (median age 71.0 years vs. 64.2 years, $\mathrm{p}<0.0001)$.

\section{5) Hospitalisation episodes}

The UKRR has done collaborative work using Hospital Episode Statistics (HES) data. This cohort included all RRT patients over the age of 18 years who started RRT for ERF in English renal centres between 1 January 2002 and 31 December 2006. Detailed methodology for this has been previously published [9]. This cohort was used to calculate unadjusted hospitalisation rates and cause of hospitalisation by ethnic group.

\section{Results}

\section{Regional variations in incidence of $R R T$}

Data completeness and ethnic composition by centre in the incident population 2003-2012 is shown in table 6.1. Overall completeness was $92 \%$, excluding Scottish centres. There was huge variation between centres in the proportion of non-White patients on
RRT in each centre, from $62 \%$ in London Barts and $55 \%$ in London West to $1 \%$ in some of the South West centres, with an overall median of $6 \%$. Ethnic distribution of the population accounted for some of the regional variations in RRT incidence. The age-gender standardized incidence ratio of RRT was higher (2-3 times) in regions with a high ethnic minority population compared to those with a low ethnic minority population (figure 6.1). However previous work by the UKRR has shown that only $31 \%$ of this regional variation in RRT incidence in the UK could be explained by demographics, health and access to health service factors [10].

\section{Age, gender and social deprivation}

$62.2 \%$ of patients were male; this degree of male preponderance was observed for White and South Asian patients although to a lesser extent with Black patients (58.0\%, $\mathrm{p}<0.0001)$ (table 6.2). The proportion of male patients amongst Black patients has however increased from the $48 \%$ observed in the 1997-2003 cohort.

Of all patients starting RRT in 2012, 49\% were aged 65 years compared to only $16 \%$ aged 65 years in the general UK population [1]. The higher incidence of RRT amongst older people was more pronounced for Black and South Asian patients compared to White patients (incidence rate of $1,191,1,133$ and 283 per million population respectively), (table 6.3, figure 6.2).

Amongst all patients starting RRT, Black and South Asians were younger compared to White patients, with median ages of 54.4, 58.7 and 65.5 years respectively $(\mathrm{p}<0.0001)($ table 6.2)

Data on residence postcode to calculate IMD score was not available for $250(0.5 \%)$ patients and this was not different between the ethnic groups. Black and South Asian patients predominantly lived in socially deprived areas. The proportion of patients living in IMD quintile 5 areas was greater for Black and South Asians than White patients $(45.7 \%, 38.7 \%, 20.9 \%$ respectively, $\mathrm{p}<0.0001)$ (table 6.4 ).

\section{Primary renal disease causing ERF}

Data on primary renal disease was missing for 2,473 $(4.9 \%)$ of all patients and this was equally distributed between the ethnic groups.

Diabetes was the leading cause of ERF in all ethnic groups. However, the proportion of patients with diabetes as cause of ERF was greater amongst South Asian and Black patients compared to White patients (40.2\%, $31.0 \%, 20.5 \%$ respectively) (table 6.5).

Amongst Black and South Asian patients diabetes was more common in those aged 65 years, as compared to 
Table 6.1. Percentage of incident RRT patients (2003-2012) in different ethnic groups by centre

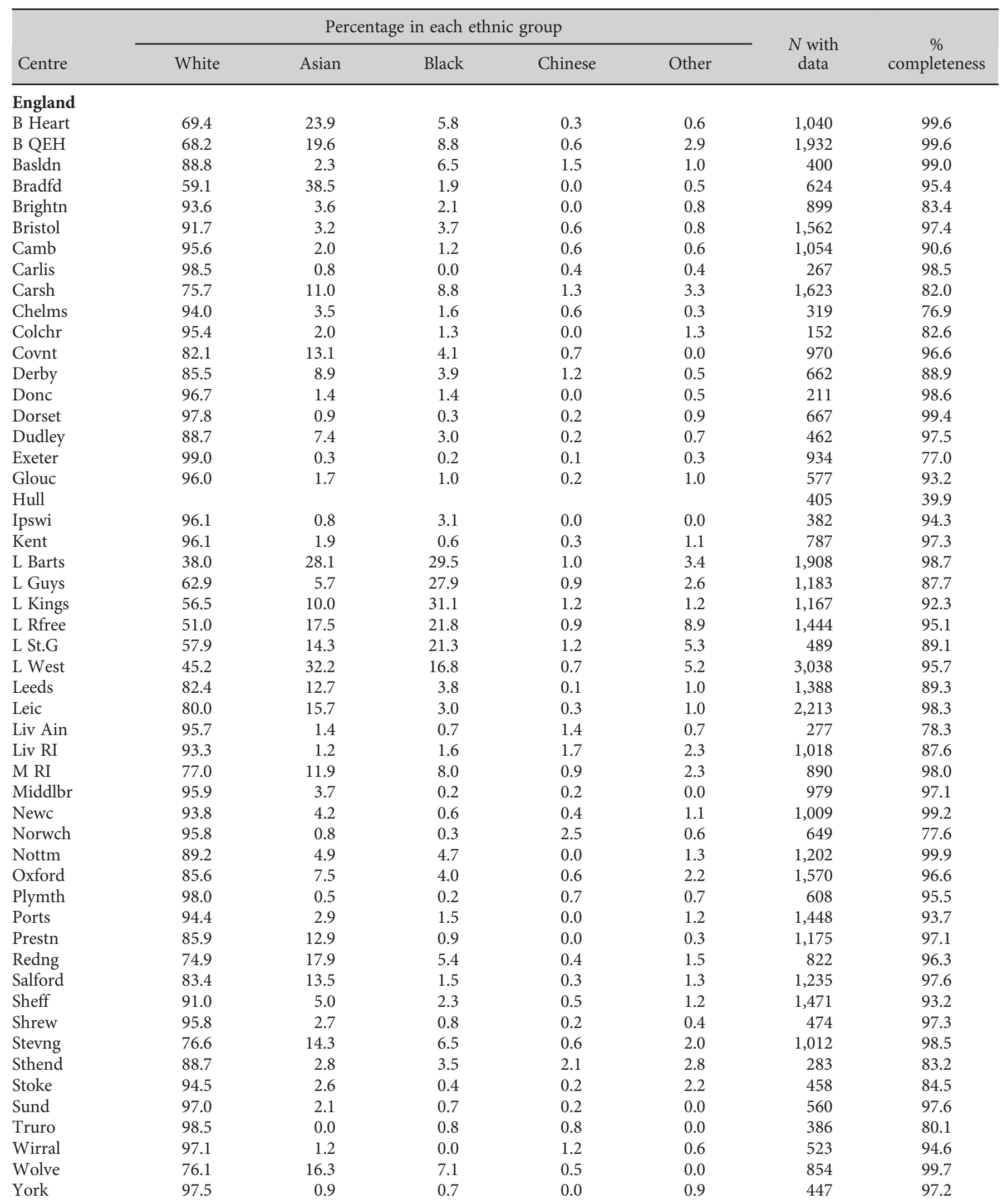


Table 6.1. Continued

\begin{tabular}{|c|c|c|c|c|c|c|c|}
\hline \multirow[b]{2}{*}{ Centre } & \multicolumn{5}{|c|}{ Percentage in each ethnic group } & \multirow{2}{*}{$\begin{array}{c}N \text { with } \\
\text { data }\end{array}$} & \multirow{2}{*}{$\begin{array}{c}\% \\
\text { completeness }\end{array}$} \\
\hline & White & Asian & Black & Chinese & Other & & \\
\hline Antrim & 98.8 & 1.2 & 0.0 & 0.0 & 0.0 & 259 & 95.6 \\
\hline Belfast & 98.4 & 0.8 & 0.2 & 0.5 & 0.3 & 665 & 94.9 \\
\hline Newry & 99.4 & 0.0 & 0.0 & 0.0 & 0.6 & 166 & 96.0 \\
\hline \multicolumn{8}{|l|}{ Scotland } \\
\hline Abrdn & & & & & & 128 & 23.0 \\
\hline Airdrie & & & & & & 206 & 42.0 \\
\hline D \& Gall & & & & & & 10 & 5.9 \\
\hline Dundee & & & & & & 203 & 34.3 \\
\hline Dunfn & & & & & & 9 & 2.6 \\
\hline \multicolumn{8}{|l|}{ Wales } \\
\hline Bangor & 97.6 & 0.7 & 0.7 & 0.4 & 0.7 & 286 & 89.1 \\
\hline Cardff & 94.1 & 4.5 & 0.9 & 0.4 & 0.2 & 1,344 & 74.1 \\
\hline Clwyd & 98.3 & 1.7 & 0.0 & 0.0 & 0.0 & 117 & 61.9 \\
\hline Swanse & 98.1 & 1.1 & 0.7 & 0.0 & 0.1 & 1,161 & 98.9 \\
\hline Wrexm & 98.3 & 0.9 & 0.4 & 0.0 & 0.4 & 230 & 83.0 \\
\hline England & 79.5 & 11.0 & 7.1 & 0.6 & 1.8 & 48,109 & 92.5 \\
\hline N Ireland & 98.4 & 1.0 & 0.1 & 0.3 & 0.2 & 1,471 & 96.1 \\
\hline Scotland & & & & & & 847 & 15.3 \\
\hline Wales & 96.3 & 2.6 & 0.7 & 0.2 & 0.2 & 3,138 & 83.1 \\
\hline E, W \& NI & 81.0 & 10.2 & 6.5 & 0.6 & 1.7 & 52,718 & 91.9 \\
\hline UK & 81.3 & 10.1 & 6.4 & 0.6 & 1.6 & 53,565 & 85.2 \\
\hline
\end{tabular}

Blank cells denote $<20$ patients or $<50 \%$ data completeness

Table 6.2. Percentage distribution of gender and age at start of RRT by ethnic group in the incident population 2003-2012

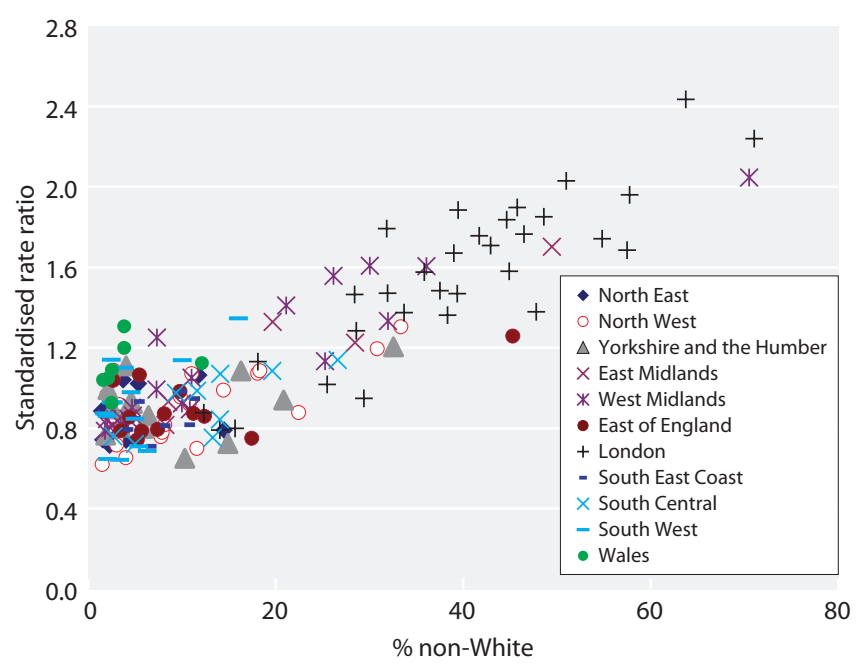

Fig. 6.1. Age/gender standardized incidence ratio (2010-2012) by percentage non-White

\begin{tabular}{lccc}
\hline & Asian & Black & White \\
\hline$N$ & 5,383 & 3,442 & 42,723 \\
$\%$ male & 61.3 & 58.3 & 62.6 \\
$\begin{array}{l}\text { Age at RRT start } \\
\text { \% }<65\end{array}$ & & & \\
\% 65+ & 64.9 & 67.6 & 48.8 \\
median & 35.1 & 32.4 & 51.2 \\
$\quad$ IQR & 58.7 & 54.4 & 65.5 \\
\hline
\end{tabular}

Table 6.3. Incidence rate by ethnic group in under 65 and over 65 year age groups at RRT start (2010-2012)

\begin{tabular}{lrrr}
\hline Incidence rate $(\mathrm{pmp})$ & Asian & Black & White \\
\hline$<65$ years & 121 & 160 & 56 \\
65 years & 1,133 & 1,191 & 283 \\
Overall & 179 & 224 & 97 \\
\hline
\end{tabular}

Pmp $=$ per million population 


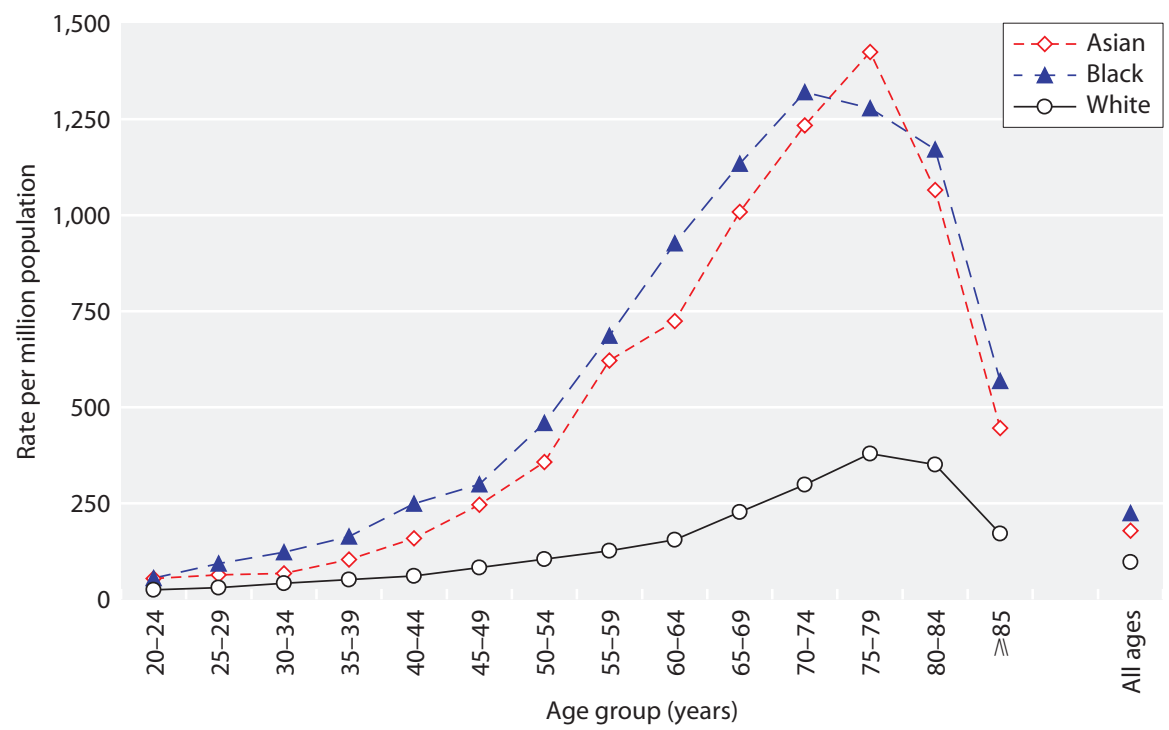

Fig. 6.2. Age profile of incident RRT patients (2010-2012), by ethnicity, in England and Wales
White patients where it was proportionally higher in those aged $<65$ years. This may reflect a difference between ethnic groups in the underlying type of diabetes leading to ERF. Adult polycystic kidney disease and renovascular disease accounted for a lower proportion of renal disease in the ethnic minority groups compared

Table 6.4. Percentage distribution of deprivation by ethnic group in incident patients 2003-2012

\begin{tabular}{lrrr}
\hline Deprivation quintile* $(\%)$ & Asian & Black & White \\
\hline 1 & 7.8 & 4.2 & 17.3 \\
2 & 10.1 & 6.4 & 19.9 \\
3 & 17.0 & 12.5 & 21.0 \\
4 & 26.5 & 31.3 & 21.0 \\
$5^{*}$ & 38.7 & 45.7 & 20.9 \\
\hline
\end{tabular}

*Quintile 5 most deprived with White patients whilst hypertensive renal disease was more common amongst Black patients.

\section{Comorbidity}

Patients with missing data on comorbidity $(N=21,896,42 \%)$ were excluded from the analyses. Data incompleteness was comparable between ethnic groups $(p=0.5)$. The results presented here should be interpreted with caution due to significant missing data. There was a wide variation in data completeness on comorbidity between centres. Results from analyses including only centres with data completeness of $50 \%$ were similar.

Overall, the proportion of patients with at least one comorbidity was greater amongst White patients compared to South Asians and Black patients (55.5\%, $45.8 \%, \quad 37.1 \%$ respectively, $\mathrm{p}<0.0001)$. However

Table 6.5. Percentage distribution of primary renal diagnosis, by ethnic group, in the incident cohort 2003-2012

\begin{tabular}{|c|c|c|c|c|c|c|c|c|c|}
\hline Diagnosis & \multicolumn{3}{|c|}{ Asian } & \multicolumn{3}{|c|}{ Black } & \multicolumn{3}{|c|}{ White } \\
\hline Uncertain aetiology & 19.7 & 23.9 & 21.2 & 15.6 & 14.2 & 15.2 & 12.6 & 24.6 & 18.7 \\
\hline Other & 13.1 & 7.3 & 11.0 & 18.4 & 10.5 & 15.9 & 17.8 & 16.4 & 17.1 \\
\hline Glomerulonephritis & 14.4 & 4.8 & 11.0 & 15.1 & 5.6 & 12.0 & 17.3 & 10.0 & 13.6 \\
\hline Renovascular disease & 1.7 & 4.6 & 2.7 & 1.5 & 4.6 & 2.5 & 2.4 & 13.2 & 7.9 \\
\hline Hypertension & 5.1 & 5.7 & 5.3 & 17.7 & 13.2 & 16.3 & 4.6 & 7.3 & 6.0 \\
\hline$N$ with data & 3,279 & 1,765 & 5,044 & 2,186 & 1,036 & 3,222 & 19,201 & 19,965 & 39,166 \\
\hline$\%$ data not available* & 4.9 & 5.3 & 5.1 & 5.4 & 6.6 & 5.8 & 4.2 & 5.5 & 4.9 \\
\hline
\end{tabular}

*This includes data not sent and data from centres excluded from analysis because $50 \%$ PRD of uncertain aetiology 
Table 6.6. Percentage of patients with comorbidity at start of RRT (2003-2012) by ethnic origin

\begin{tabular}{lrrr}
\hline Comorbidity & Asian & Black & White \\
\hline Coronary heart disease & 25.1 & 9.8 & 22.0 \\
Diabetes (not listed as PRD) & 9.5 & 6.4 & 8.4 \\
Diabetes (as PRD or comorbidity) & 40.0 & 30.9 & 20.0 \\
COPD* & 3.7 & 2.2 & 7.6 \\
Malignancy & 4.1 & 6.2 & 13.5 \\
Liver disease & 4.0 & 4.7 & 2.6 \\
Smoking & 6.6 & 7.3 & 15.2 \\
Vascular disease & 15.2 & 14.8 & 20.1 \\
One or more comorbidities present & 45.8 & 37.1 & 55.5 \\
\hline
\end{tabular}

${ }^{*}$ Chronic obstructive pulmonary disease

diabetes (both as primary renal disease and as a comorbidity not causing renal disease) was more common amongst the two ethnic minorities. Coronary heart disease was more common in South Asian and White patients compared to Black patients. Vascular disease, malignancy and smoking were more common amongst White patients (table 6.6). These trends were seen in both those aged $<65$ and 65 , although the magnitude of difference between the ethnic groups for the two age groups varied depending on the comorbidity (table 6.7).

\section{Late presentation}

19,817 (38.4\%) patients were excluded from the analysis due to not having data on the date first seen by a nephrologist. Overall, late referral has decreased over the years with the majority (64\%) of patients being referred at least a year or more prior to start of RRT compared to only $46 \%$ in 1997-2003, although one should interpret this with caution due to potential bias introduced by the significant proportion of missing data. This overall decrease in late referral compared to the previous cohort years was observed in all ethnic groups. However, late referral was more common amongst White patients compared to Black and South Asian patients (21.3\%, $19.9 \%, 17.6 \%$ respectively, $\mathrm{p}<0.0001)$. There was an age interaction with referral pattern between ethnic groups in that late referral was more common amongst White patients but only in those aged 65 (table 6.8). When stratified by diabetic status, there was no difference in late referral between ethnic groups (table 6.9). This suggests that the early referral patterns observed in Black and South Asian patients was probably due to higher incidence of diabetes in these groups.

\section{Treatment modality}

Haemodialysis (HD) was the commonest starting RRT modality in all ethnic groups (73.3\%) followed by peritoneal dialysis (PD) (21.2\%) and pre-emptive transplantation (5.6\%). The proportion of patients starting PD was lower amongst Black and South Asians compared to White patients $(16.4 \%, 18.4 \%, 21.9 \%$ respectively, $\mathrm{p}<0.0001)$. Similarly, pre-emptive transplantation rates were lower amongst South Asian and Black patients compared to White patients $(3.1 \%, 4.2 \%, 6.0 \%$ respectively, $\mathrm{p}<0.0001)$. There was no difference $(\mathrm{p}=0.6)$ in the type of kidney donor (post circulatory death donor, post brain stem death donor, live donor) between the ethnic groups amongst those who had a pre-emptive kidney transplant. Compared to those referred late ( $<90$ days of RRT start), patients who were referred earlier were more likely to start on PD $(25.0 \%$ vs. $11.2 \%, \mathrm{p}<0.0001)$ and had more pre-emptively transplantation $(6.9 \%$ vs. $1.0 \%, \mathrm{p}<0.0001)$. This trend was seen in all ethnic groups except in Black patients where the pre-emptive transplantation rate was similar amongst those referred early and late (data not shown).

Table 6.7. Percentage of patients with comorbidity at start of RRT (2003-2012) by age and ethnic origin

\begin{tabular}{|c|c|c|c|c|c|c|}
\hline \multirow[b]{2}{*}{ Comorbidity } & \multicolumn{2}{|c|}{ Asian } & \multicolumn{2}{|c|}{ Black } & \multicolumn{2}{|c|}{ White } \\
\hline & $<65$ & $65+$ & $<65$ & $65+$ & $<65$ & $65+$ \\
\hline Coronary heart disease & 19.1 & 37.1 & 5.7 & 18.9 & 13.4 & 30.2 \\
\hline Diabetes (not listed as PRD) & 7.4 & 13.9 & 5.1 & 9.1 & 5.0 & 11.7 \\
\hline Diabetes (as PRD or comorbidity) & 35.9 & 47.6 & 23.7 & 46.2 & 23.7 & 16.6 \\
\hline $\mathrm{COPD}^{*}$ & 3.0 & 5.1 & 1.4 & 4.0 & 4.8 & 10.2 \\
\hline Malignancy & 2.4 & 7.6 & 3.9 & 11.4 & 7.5 & 19.2 \\
\hline Liver disease & 3.9 & 4.1 & 5.3 & 3.2 & 3.4 & 1.7 \\
\hline Smoking & 7.3 & 5.3 & 8.2 & 5.2 & 18.1 & 12.5 \\
\hline Vascular disease & 11.6 & 22.7 & 10.1 & 25.1 & 14.7 & 25.2 \\
\hline One or more comorbidities present & 37.4 & 62.8 & 30.0 & 52.4 & 44.4 & 66.2 \\
\hline
\end{tabular}

${ }^{*}$ Chronic obstructive pulmonary disease 
Table 6.8. Presentation in incident patients $2003-2012$, by ethnicity and age

\begin{tabular}{|c|c|c|c|c|c|c|}
\hline \multirow[b]{2}{*}{ Presentation } & \multicolumn{2}{|c|}{ Asian } & \multicolumn{2}{|c|}{ Black } & \multicolumn{2}{|c|}{ White } \\
\hline & $<65$ & $65+$ & $<65$ & $65+$ & $<65$ & $65+$ \\
\hline$N$ & 1,822 & 935 & 955 & 471 & 13,425 & 14,123 \\
\hline$\%<90$ days & 18.8 & 15.7 & 22.1 & 14.7 & 19.9 & 22.6 \\
\hline$\% 90-365$ days & 16.7 & 12.8 & 16.7 & 11.9 & 15.9 & 14.3 \\
\hline$\%>365$ days & 64.5 & 71.4 & 61.3 & 73.5 & 64.2 & 63.1 \\
\hline
\end{tabular}

\section{eGFR at start of RRT}

The eGFR at start of RRT has increased over the years indicating patients are being started on RRT earlier in the course of their chronic kidney disease stage (CKD) (figure 6.3). This trend was observed in all ethnic groups. White patients started at a higher eGFR compared to Black and South Asian patients. The median eGFR at RRT start in 2003-2012 for White patients was $8.5 \mathrm{ml} / \mathrm{min} / 1.73 \mathrm{~m}^{2}$ compared to $8.0 \mathrm{ml} / \mathrm{min} / 1.73 \mathrm{~m}^{2}$ for Black and $7.8 \mathrm{ml} / \mathrm{min} / 1.73 \mathrm{~m}^{2}$ for South Asian patients $(\mathrm{p}<0.0001)$. As missing data accounted for $49 \%$ of this cohort, caution should be taken in interpreting this result.

Preliminary work undertaken by the UKRR on a cohort of CKD stage 5 patients in the UK has shown that Black and South Asian patients had a much more rapid decline in their eGFR in the year preceding RRT compared to White patients despite adjustments for age, gender and primary renal disease (unpublished data).

\section{Haemoglobin prior to start of RRT}

Due to missing data, 25,134 (49\%) patients were excluded. White patients had higher mean $\mathrm{Hb}(102.3 \mathrm{~g} / \mathrm{L})$ prior to start of RRT compared to South Asian patients $(99.9 \mathrm{~g} / \mathrm{L}, \mathrm{p}<0.0001)$ and Black patients $(95.7 \mathrm{~g} / \mathrm{L}$, $\mathrm{p}<0.0001)$. Data on erythropoietin use prior to start of RRT was not available to further explore the reasons for the differences in $\mathrm{Hb}$ at start of RRT between ethnic groups. As it is well known that diabetic patients (more common amongst Black and South Asian patients) become more anaemic earlier in their CKD course compared to non-diabetics [11], a stratified analysis by diabetes status was performed but the results were similar (data not shown).

\section{Patient outcome measures}

Attainment of laboratory standards on dialysis

The proportion of patients in each ethnic group who achieved the Renal Association standard varied depending on the outcome measure studied. Table 6.10 shows the multivariate logistic regression model with and without adjustments for various confounding factors. Compared to White patients, South Asian patients had similar attainment of the $\mathrm{Hb}$ and $\mathrm{PTH}$ standards; better attainment for the URR and phosphate standards; and lower attainment of the calcium standard. Black patients had similar attainment of the $\mathrm{Hb}$, calcium and PTH standards; lower attainment of the URR standard but better attainment of the phosphate standard.

\section{Access to kidney transplantation}

The UKRR in collaboration with the Organ Donation Transplantation Directorate of NHS Blood and Transplant (ODT) previously reported on access to kidney transplantation for the ethnic minority patients starting RRT in the years 1997-2004 [5, 6, 7]. Compared to the White patients, South Asian (hazard ratio (HR) 1.10, 95\% CI 0.97-1.24) and Black patients (HR 0.95, 95\% CI. 0.79-1.14) had similar rates of being listed for a kidney transplant once adjusted for various patient characteristics including social deprivation and centre

Table 6.9. Presentation in incident patients 2003-2012, by ethnicity, stratified by diabetes

\begin{tabular}{|c|c|c|c|c|c|c|}
\hline \multirow[b]{2}{*}{ Presentation } & \multicolumn{2}{|c|}{ Asian } & \multicolumn{2}{|c|}{ Black } & \multicolumn{2}{|c|}{ White } \\
\hline & Diabetic & Non-diabetic & Diabetic & Non-diabetic & Diabetic & Non-diabetic \\
\hline$N$ & 1,024 & 1,584 & 403 & 937 & 5,335 & 20,394 \\
\hline$\%<90$ days & 12.1 & 21.3 & 11.9 & 21.7 & 10.3 & 23.4 \\
\hline$\%$ 90-365 days & 18.4 & 13.1 & 18.1 & 13.5 & 17.7 & 14.2 \\
\hline$\%>365$ days & 69.5 & 65.7 & 70.0 & 64.9 & 72.0 & 62.5 \\
\hline
\end{tabular}




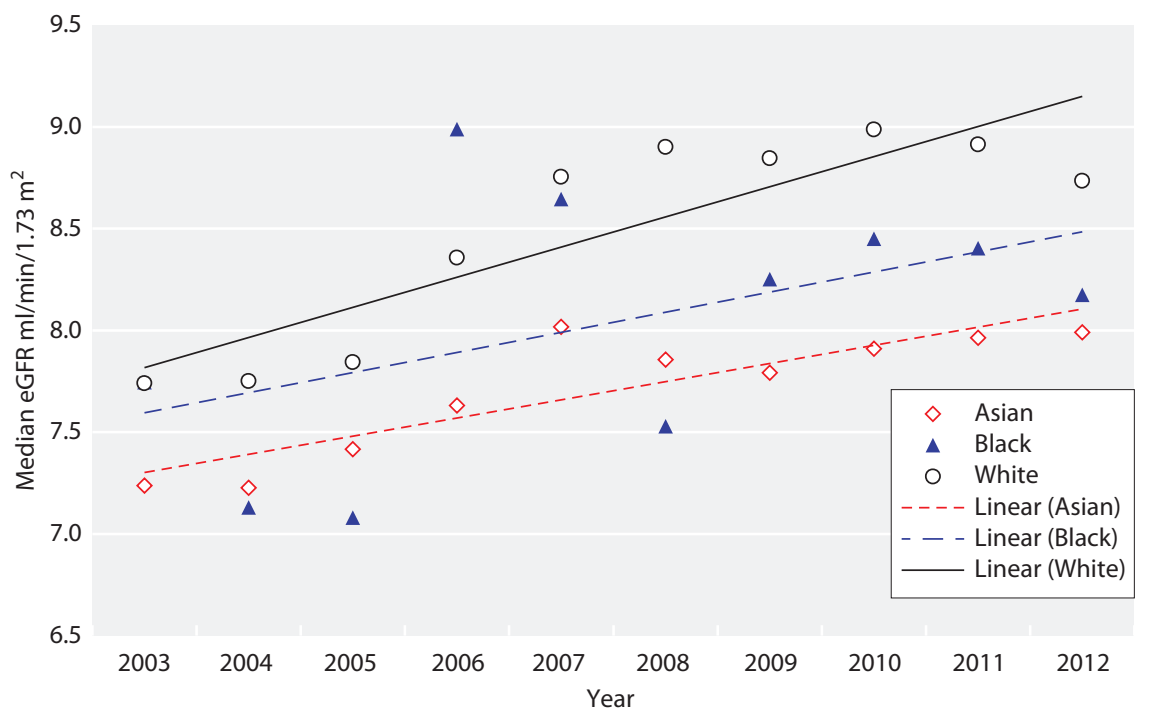

Fig. 6.3. Median eGFR at start of RRT by year of start and ethnic group effects. However, once on the waiting list, South Asian (HR $0.74,95 \%$ CI $0.65-0.85$ ) and Black patients (HR 0.66 , 95\% CI 0.49-0.87) had lower rates of deceased donor kidney transplantation. Similarly the likelihood of living donor kidney transplantation in the fully adjusted analyses was lower for South Asian patients (odds ratio (OR) 0.66, 95\% CI 0.45-0.96) and Black patients (OR $0.40,95 \%$ CI $0.21-0.73$ ) compared to White patients. A more recent analysis of patients starting RRT between 2006 and 2008 confirmed no ethnic disparities in access to waiting list but the lower rates of deceased donor transplantation once waitlisted, and for live donor transplantation persisted for the ethnic minorities [12].

\section{Kidney transplant outcomes}

One year graft outcomes

The analyses included 9,091 kidney only transplants. Of these kidney only transplants, 237 (2.5\%) were excluded either due to lack of matching between the UKRR and ODT databases $(N=159)$ or lost to follow up $(N=78)$.
Graft failure (excluding deaths with functioning grafts) in the first year following kidney transplantation was greater for Black patients (7.5\%) and South Asian (6.1\%) patients compared to White patients (4.2\%) $(\mathrm{p}=0.0001)$. However, in the multivariate Cox regression analyses censoring for death, South Asian patients had a similar graft survival but Black patients a lower graft survival compared to White patients (table 6.11). Results were similar when analyses were repeated with death as a competing risk event. Amongst those who had a functioning graft at one year post kidney transplantation $(N=8,479)$, the median eGFR was better for Black $\left(57.2 \mathrm{ml} / \mathrm{min} / 1.73 \mathrm{~m}^{2}\right.$, interquartile range (IQR) 42.971.5 ) and South Asian (58.5 ml/min/1.73 m², IQR 45.2, $73.3)$ patients compared to White $\left(51.5 \mathrm{ml} / \mathrm{min} / 1.73 \mathrm{~m}^{2}\right.$, IQR 40.0, 64.1, $\mathrm{p}<0.0001)$ patients.

Five year graft outcomes

For the analyses, 2,912 kidney only transplants were included. Of these kidney only transplants, 126 (4.1\%) were excluded either due to lack of matching between

Table 6.10. Odds ratio (OR) (95\% confidence interval) of attainment of RA standards at one year after starting RRT in dialysis patients, in Asian and Black patients compared to White patients

\begin{tabular}{|c|c|c|c|c|c|c|c|c|}
\hline & \multicolumn{2}{|c|}{ White } & \multicolumn{3}{|c|}{ Asian } & \multicolumn{3}{|c|}{ Black } \\
\hline & OR & $N$ & Unadjusted & Adjusted & $N$ & Unadjusted & Adjusted & $N$ \\
\hline Haemoglobin & 1 & 23,982 & $1.01(0.94-1.09)$ & $1.03(0.94-1.11)$ & 3,255 & $0.98(0.90-1.07)$ & $0.94(0.85-1.04)$ & 2,135 \\
\hline Calcium & 1 & 21,375 & $0.85(0.79-0.92)$ & $0.89(0.81-0.97)$ & 3,018 & $0.86(0.78-0.94)$ & $0.95(0.85-1.06)$ & 2,023 \\
\hline Phosphate & 1 & 23,559 & $1.03(0.96-1.11)$ & $1.15(1.06-1.25)$ & 3,221 & $1.05(0.96-1.15)$ & $1.21(1.09-1.34)$ & 2,114 \\
\hline PTH & 1 & 20,553 & $1.12(1.04-1.21)$ & $1.05(0.96-1.15)$ & 2,685 & $1.05(0.95-1.15)$ & $0.97(0.87-1.09)$ & 1,737 \\
\hline URR & 1 & 14,393 & $1.62(1.42-1.84)$ & $1.73(1.49-2.00)$ & 1,961 & $0.81(0.70-0.93)$ & $0.77(0.65-0.91)$ & 1,011 \\
\hline
\end{tabular}


Table 6.11. Cox-regression analysis of one year graft failure by ethnicity of kidney-only transplants between 2003 and 2011

\begin{tabular}{llllll}
\hline & \multicolumn{2}{c}{ Unadjusted Cox-regression } & & \multicolumn{2}{c}{ Adjusted Cox-regression } \\
\cline { 2 - 3 } \cline { 5 - 6 } & HR $(95 \% \mathrm{CI})$ & $\mathrm{p}$-value & & HR (95\% CI) & 0.1 \\
\hline Asian & $1.4(1.1-1.9)$ & 0.01 & & $1.3(0.9-1.9)$ & 0.0007 \\
Black & $1.8(1.3-2.5)$ & 0.0004 & & 1 (reference) & \\
White & 1 (reference) & & & \\
\hline
\end{tabular}

the UKRR and ODT databases $(N=101)$ or lost to follow up $(N=25)$. Graft failure (excluding deaths with functioning grafts) at five years following kidney transplantation was greater for Black patients (17.2\%) compared to South Asian (9.2\%) and White $(9.8 \%)(\mathrm{p}=0.03)$ patients. In the multivariate Cox regression analyses censoring for death, White and South Asian patients had a similar graft survival but Black patients had lower graft survival (table 6.12). Results were similar when analyses were repeated with death as a competing risk event. Amongst those who had a functioning graft at five years post kidney transplantation $(N=2,482)$, the median eGFR was better for Black $\left(60.4 \mathrm{ml} / \mathrm{min} / 1.73 \mathrm{~m}^{2}\right.$, IQR 42.8-75.7) and South Asian $\left(58.1 \mathrm{ml} / \mathrm{min} / 1.73 \mathrm{~m}^{2}\right.$, IQR $44.7,71.3)$ patients compared to White patients $(50.3 \mathrm{ml} /$ $\min / 1.73 \mathrm{~m}^{2}$, IQR 38.0, 64.2, $\left.\mathrm{p}<0.0001\right)$.

\section{Patient survival}

Figure 6.4 shows the unadjusted survival in the first year of RRT for the different age groups. Overall, South Asian and Black patients have better survival than White patients and this is more apparent in the 55-75 age groups. The survival of patients on RRT in the first year has improved over the years 2003-2011 for both South Asian and White patients but there appears to be a declining trend for Black patients (figure 6.5). In the multivariate adjusted Cox regression analysis including 41920 patients, survival after 90 days of starting RRT without censoring for transplantation was better for South Asian and Black patients compared to White patients (table 6.13). Results were similar when censored for transplantation (data not shown).
Deaths due to cerebrovascular disease, ischemic heart disease and infection were more common for South Asian and Black patients, whilst deaths due to malignancy, withdrawal from RRT and other causes were more common in White patients. These trends were seen both in those aged $<65$ and 65 years (table 6.14).

\section{Hospitalisation episodes}

The number of admissions and the number of admitted days per year was greater for HD patients compared to PD patients. Amongst HD patients, the number of admissions and the number of admitted days per year was greater for White patients compared to South Asian and Black patients $(\mathrm{p}<0.001)$; for PD patients, there was no major difference seen between the ethnic groups (unpublished data). The reasons for admission for the ethnic groups are shown in table 6.15. Cautious interpretation from these data is required as a significant proportion of patients had 'CKD not otherwise specified' coded as a reason for the hospitalisation.

\section{Discussion}

Data completeness on ethnicity has improved over the most recent years reducing the probability of selection bias that might have occurred due to missing ethnicity data in the previous years' reports. Therefore, one should interpret with caution any perceived time trends in incidence rates or patient demographics between ethnic groups.

Table 6.12. Cox-regression analysis of five year graft failure by ethnicity of kidney-only transplants between 2003 and 2007

\begin{tabular}{llclll}
\hline & \multicolumn{2}{c}{ Unadjusted Cox-regression } & & \multicolumn{2}{c}{ Adjusted Cox-regression } \\
\cline { 2 - 3 } \cline { 5 - 6 } & HR $(95 \% \mathrm{CI})$ & p-value & & HR $(95 \% \mathrm{CI})$ & $0.9(0.6-1.4)$ \\
Asian & $0.9(0.6-1.5)$ & 0.8 & & $1.5(1.1-2.1)$ & 0.6 \\
Black & $1.8(1.1-2.8)$ & 0.01 & & 1 (reference) & 0.02 \\
White & 1 (reference) & & & \\
\hline
\end{tabular}



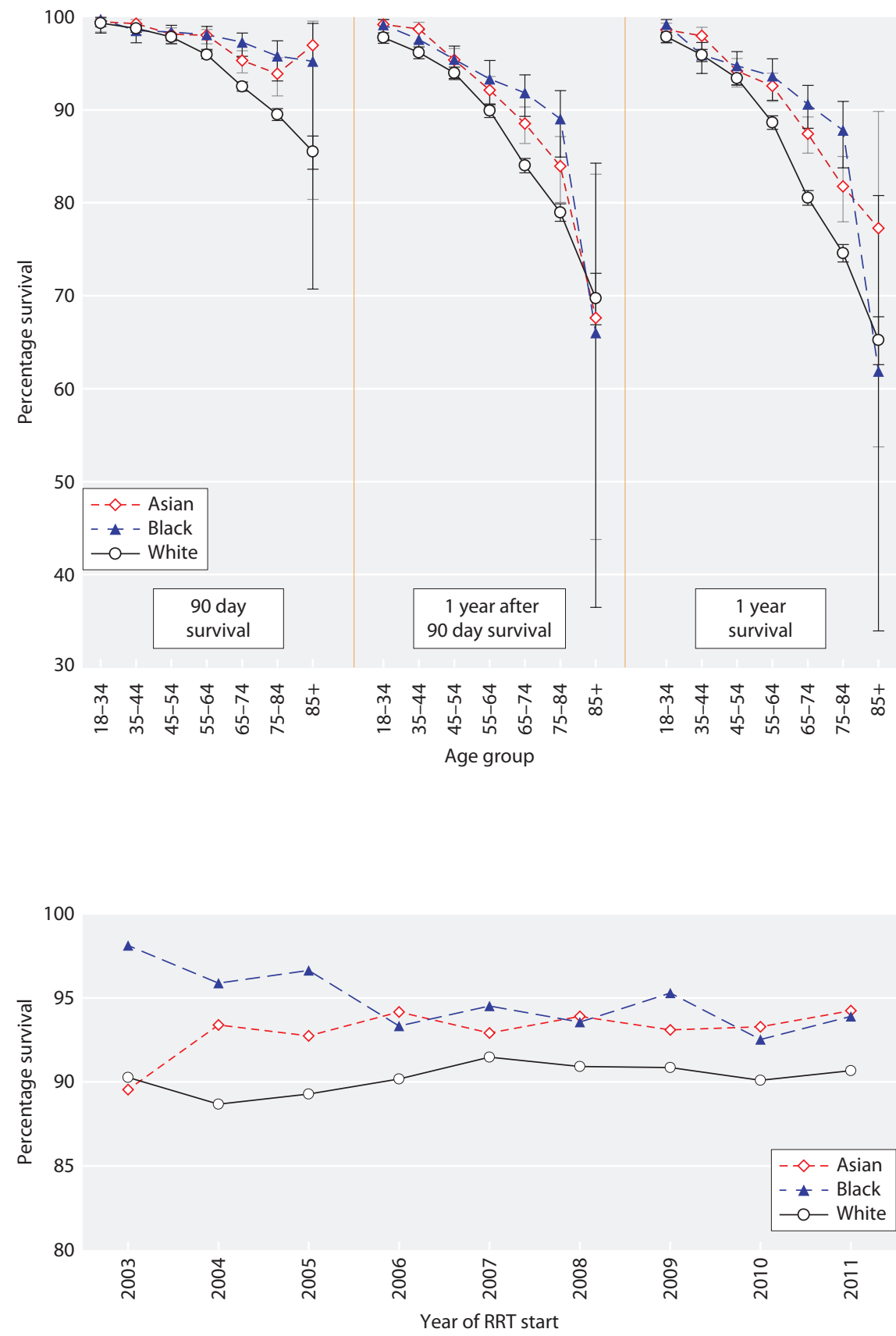

Fig. 6.4. Unadjusted survival by age group and ethnicity in patients starting RRT between 2003 and 2012
Fig. 6.5. Age- 60 adjusted survival one year after 90 days of incident patients by year of RRT start and ethnic group

Table 6.13. Cox-regression analysis of patient survival after 90 days from RRT start, by ethnic group, incident cohort $2003-2012$

\begin{tabular}{|c|c|c|c|c|}
\hline & \multicolumn{2}{|c|}{ Unadjusted Cox-regression } & \multicolumn{2}{|c|}{ Adjusted Cox-regression } \\
\hline & HR (95\% CI) & $\mathrm{p}$-value & HR (95\% CI) & p-value \\
\hline Black & $0.5 \quad(0.46-0.54)$ & $<0.0001$ & $0.58(0.52-0.64)$ & $<0.0001$ \\
\hline White & 1 (reference) & & 1 (reference) & \\
\hline
\end{tabular}


Table 6.14. Cause of deaths for incident patients 2003-2012 that died by the end of 2012, by ethnic group

\begin{tabular}{|c|c|c|c|c|c|c|c|c|c|}
\hline & \multicolumn{3}{|c|}{ All ages } & \multicolumn{3}{|c|}{ Age $<65$} & \multicolumn{3}{|c|}{ Age 65} \\
\hline & Asian & Black & White & Asian & Black & White & Asian & Black & White \\
\hline$\%$ of incident patients & 27.4 & 22.9 & 40.9 & 18.9 & 15.4 & 24.0 & 43.2 & 38.5 & 57.0 \\
\hline \multicolumn{10}{|l|}{ COD (\%) } \\
\hline Cerebrovascular disease & 6.7 & 8.4 & 3.9 & 7.2 & 8.3 & 3.4 & 6.3 & 8.5 & 4.0 \\
\hline Malignancy & 6.4 & 7.2 & 9.5 & 5.9 & 6.2 & 10.7 & 6.9 & 8.1 & 9.1 \\
\hline Other & 17.4 & 16.3 & 24.6 & 18.7 & 21.2 & 27.5 & 16.3 & 11.9 & 23.4 \\
\hline Treatment withdrawal & 9.4 & 12.1 & 17.1 & 5.3 & 7.3 & 10.0 & 12.7 & 16.6 & 19.8 \\
\hline Uncertain & 8.1 & 10.6 & 5.6 & 9.1 & 8.3 & 5.4 & 7.4 & 12.8 & 5.7 \\
\hline$N$ with no COD data & 654 & 384 & 7,984 & 286 & 166 & 2,348 & 368 & 218 & 5,636 \\
\hline
\end{tabular}

$\mathrm{COD}=$ cause of death

Black and South Asian patients were younger compared to White patients. This, to a certain extent, was probably a reflection of the younger age distribution for ethnic minorities in the general population with only

Table 6.15. Cause of hospitalisation from 90 days to one year following the start of dialysis amongst incident patients between 2002-2006, by ethnic group

\begin{tabular}{lrrr}
\hline & \multicolumn{3}{c}{ Percentage } \\
\cline { 2 - 4 } Cause of hospitalisation & Asian & Black & White \\
\hline Abdominal pain & 2.7 & 1.9 & 1.7 \\
Access & 19.6 & 23.3 & 17.9 \\
Biochemistry & 1.2 & 2.4 & 1.5 \\
Bronchitis & 4.7 & 3.2 & 3.6 \\
Cancer & 0.8 & 1.2 & 2.2 \\
Catheter & 1.0 & 1.3 & 1.6 \\
Chest pain & 2.7 & 1.4 & 1.6 \\
CKD codes & 32.5 & 33.3 & 34.1 \\
CVA & 0.7 & 0.7 & 0.7 \\
Fracture & 1.8 & 1.3 & 2.5 \\
Gastroenteritis & 3.7 & 2.6 & 3.4 \\
GI bleed & 0.3 & 0.4 & 0.8 \\
Hernia & 0.4 & 0.6 & 0.9 \\
High risk sepsis & 3.3 & 3.2 & 3.3 \\
Ischaemic heart disease & 6.3 & 3.7 & 5.9 \\
Low risk sepsis & 2.9 & 2.0 & 1.8 \\
Miscellaneous & 6.7 & 8.9 & 7.3 \\
Neuro & 1.9 & 2.2 & 1.9 \\
Overload & 2.5 & 2.6 & 2.4 \\
Peritonitis & 1.1 & 1.5 & 1.2 \\
Syncope & 1.6 & 1.4 & 2.0 \\
UTI & 1.7 & 0.8 & 1.7 \\
Total numbers & $\mathbf{1 , 9 8 9}$ & $\mathbf{1 , 8 0 2}$ & $\mathbf{2 8 , 1 0 4}$ \\
\hline CVA cerebrovasculary accident & & &
\end{tabular}

$\mathrm{CVA}=$ cerebrovascular accident

$\mathrm{UTI}=$ urinary tract infection
$6 \%$ of Black and South Asian patients being aged 65 years compared to $18 \%$ of White patients [1]. It is well established that the progression to ERF and the incidence of RRT is much greater amongst ethnic minorities compared to Whites [13-18]. However, these analyses showed that the disparity in incidence rates was more pronounced amongst those aged 65 years and the reasons for this are not obvious.

Life expectancy estimates for ethnic minorities in the general population are lower than for the White population [19] and therefore the higher incidence amongst the elderly ethnic minority patients cannot be attributed to the possibility of them living longer to reach ERF. It is also not known if there are variations in the uptake of conservative management of ERF between the ethnic groups. Although the incidence of RRT (supply) is higher in the ethnic minorities, population estimates of CKD stage 5 (demand) are needed to ensure that there is no ethnic disparity in access to RRT (demand-supply mismatch).

The proportion of patients starting RRT who had at least one comorbidity was greater amongst White patients although ill-health is generally more frequently reported by ethnic minorities in the general population [20]. However, the comorbidity patterns in the RRT population are consistent with greater incidence of coronary heart disease in South Asian patients, cerebrovascular accidents in Black patients and lower cancer rates seen in ethnic minorities in the general population [20].

Early referral to a renal centre was associated with better uptake of PD. However despite being referred earlier, ethnic minorities had lower uptake of PD and lower $\mathrm{Hb}$ at start of RRT. They also started RRT at a 
lower eGFR compared to White patients. The lower uptake of PD seen in ethnic minorities may however be as a consequence of confounding by differing centre practices of $\mathrm{PD}$ use. It is also possible that the unexpected rapid decline in kidney function in the preceding year of RRT (unpublished work by UKRR) could have resulted in insufficient time for adequate education about dialysis modalities to enable patients to choose PD, or the appropriate management of anaemia prior to the need for RRT.

However, once established on dialysis, the attainment of laboratory standards was better or similar for the ethnic minorities for most standards except calcium for South Asian and URR for Black patients. Importantly, the attainment of the $\mathrm{Hb}$ standard (which was lower at start of RRT) was no longer different between the ethnic groups at one year from start of RRT. Data on use of calcium containing phosphate binders, vitamin D analogues, duration of HD session and type of vascular access are not available to explore the reasons for these differences. These results are slightly different from those previously reported [21] on a cohort of patients starting RRT between 1997-2004 in which attainment of $\mathrm{Hb} 100 \mathrm{~g} / \mathrm{L}$ was lower amongst Black patients and attainment of PTH $32 \mathrm{pmol} / \mathrm{L}$ was lower for South Asian and Black patients. These differences were probably due to the different range used for each of the laboratory measures analysed in this report to comply with current UK guidelines. When analyses were repeated using the previous RA standards, results were similar to the earlier report.

It is reassuring to note equitable access to the transplant waiting list for ethnic minorities but there continues to be a disparity in access to deceased donor transplantation once on the waiting list. It is well acknowledged that this is due to blood group and HLA disparity compared with the predominantly White donor pool in the UK. The new UK organ allocation scheme introduced in 2006 gave a greater emphasis in the points scoring system to patients waiting longer for a transplant. The lack of observed impact in this report following the introduction of the new scheme may be due to the fact that the majority of patients included in this report irrespective of their ethnicity would have waited for a similar duration of time on the waiting list, whereas the new allocation scheme would have improved access to a small proportion who were on the waiting list well before 2006. Living donor transplantation rates were lower for ethnic minorities and several recipient and donor factors have been suggested including fewer

Demographics and outcomes of patients by ethnic group approaches or less active encouragement by nephrologists to seek living related donors [22]; lack of suitable donors with family members living outside the UK who are therefore unable to be assessed or complete donor work up; and high prevalence of diabetes in the immediate family [23]. It has also been observed that Black patients on dialysis had more positive coping strategies than Whites and this may affect their perception of the need for transplant [24].

The poor graft survival for Black patients reported in this cohort is consistent with previous reports from the UK $[25,26]$ and the USA $[27,28]$. However a study from France suggested that compared to White patients, graft survival was similar for Black patients with a genetic pool similar to African Americans suggesting the possible role of social deprivation and health care access in poor outcomes for Black patients in the USA [29]. In the analyses, these disparities were observed despite adjustments for area level deprivation. Black and Indo Asian patients have a greater likelihood of receiving kidneys at higher risk of delayed or inferior outcomes, i.e. expanded criteria donor (ECD) kidneys, compared to White patients in the USA [30]. Previous UKRR work in collaboration with ODT has shown that Black and South Asian patients were more likely to receive kidneys with longer cold ischaemic time and HLA mismatches both of which could influence graft survival [7]. Donor information for this cohort was not available to explore the reasons for the apparent persistent inferior graft survival for Black patients in the UK.

There was a paradox in that Black and South Asian patients despite having reduced life expectancy in the general population [19] appeared to have better survival on dialysis. No adjustment for baseline comorbidity was made in this report due to incomplete data but these results are consistent with previous studies from North America and the UK that have adjusted for baseline comorbidity although residual confounding from missing comorbidity data could not be excluded in these studies $[31,32,33$.

Hospitalisation rates were higher for White patients on dialysis compared to South Asian and Black patients. Due to several of these episodes being coded as 'CKD not otherwise specified', it was not possible to determine if the increased hospitalisation rates amongst White dialysis patients was due to newly acquired comorbidity whilst on RRT that could account for the increased mortality. Several mechanisms including better adaptation on dialysis, better social support, less withdrawal from dialysis and greater use of Vitamin D analogues amongst ethnic minorities have also been suggested for 
better survival amongst ethnic minority dialysis patients $[34,35,36,37]$. Another possible mechanism suggested for this paradox is survivor bias i.e. ethnic minority patients with CKD and significant comorbidity are more likely to die prematurely before reaching ERF or possibly less likely to be referred or accepted onto RRT [38]. However a more recent study from the USA has shown that mortality is similar between Black and White patients with CKD stages 3-4 questioning this hypothesis [39].

Another possible mechanism is lead time bias. White patients started RRT at a slightly higher eGFR compared to ethnic minorities in this study. However, this difference was clinically very small to entirely account for the ethnic differences in mortality observed in this study. It is well established that Black and South Asian patients have rapid progression from their underlying CKD to ERF. It is therefore possible that they have less 'CKD vintage' compared to the White patients i.e may therefore start RRT early with a reduced arteriosclerotic load when compared with the White population. Although ischaemic heart disease was more common amongst South Asian patients, the proportion of patients with at least one comorbidity and those with vascular disease and smoking were more prevalent in White patients.
Further studies examining survival from a predefined eGFR early in the course of CKD stage $4-5$ are needed to explore this hypothesis with more detailed assessment of CVD (e.g. LVEF, ABPI etc.).

There are other patient outcome measures that merit comparison between ethnic groups on RRT in the UK such as quality of life and mental health. This is currently within the remit of collaborative work being considered by the UKRR. Data on cause of hospitalisation episodes for dialysis patients are required to help understand the differences in survival between the ethnic groups.

This report confirms the persistent high incidence of RRT, the better survival on dialysis and the poor access to kidney transplantation for South Asian and Black patients and early allograft loss for Black patients.

This, in the context of increasing ethnic diversity of the general population and ageing of ethnic minorities will have a significant impact on the prevalence of ethnic minority patients on dialysis and impose a disproportionate demand on dialysis provision in those areas with a high ethnic minority population. More effort is needed to reduce progression of $\mathrm{CKD}$ to $\mathrm{ERF}$ in ethnic minorities.

Conflicts of interest: none

\section{References}

1 Office for National Statistics, 2011 Census: Aggregate data (England and Wales). UK Data Service Census Support

-2 Shaw C et al. UK Renal Registry 14th annual report: Chapter 4 comorbidities and current smoking status amongst patients starting renal replacement therapy in England, Wales and Northern Ireland from 2009 to 2010. Nephron Clin Pract 2012;120(suppl 1):c81-c91

-3 Payne RA and GA Abel. UK indices of multiple deprivation - a way to make comparisons across constituent countries easier. Health Statistics Quarterly 53, Spring 2012. Office of National statistics. London

4 Levey AS et al. A simplified equation to predict glomerular filtration rate from serum creatinine [abstract]. J Am Soc Nephrol 2000;11:A0828

5 Udayaraj et al. Social deprivation, ethnicity, and uptake of living kidney donor transplantation in the United Kingdom. Transplantation 2012; Mar 27;93(6)

6 Udayaraj et al. Social deprivation, ethnicity, and access to the deceased donor kidney transplant waiting list in England and Wales. Transplantation 2010 Aug 15;90(3):279-285

7 Udayaraj et al. Social deprivation, ethnicity and renal replacement therapy in England and Wales: Equity of access and outcomes (Dissertation). University of Bristol, England, 2009

8 Levey A, Stevens L, Schimd C, et al. A new equation to estimate glomerular filtration rate. Ann Intern Med 2009;150(9):604-612

-9 Fotheringham J et al. Variation in centre-specific survival in patients starting renal replacement therapy in England is explained by enhanced comorbidity information from hospitalization data. Nephrol Dial Transplant (2013) 0:doi: 10.1093/ndt/gft363

10 Castledine et al. How much of the regional variation in RRT incidence rates within the UK is explained by the health needs of the general population? Nephrol Dial Transplant 2012 Oct;27(10):3943-3950
11 Anemia management in people with chronic kidney disease. National Institute for Health and Care Excellence 2011. Downloaded from www.nice.org.uk

12 Pruthi R et al. UK Renal Registry 15th annual report: Chapter 9 centre variation in access to renal transplantation in the UK (2006-2008). Nephron Clin Pract 2013:123(suppl 1):183-193

13 Department of Health: Saving lives: Our healthier nation. London: HMSO. 1999

14 Bryson CL, Ross HJ, Boyko EJ, Young BA. Racial and ethnic variations in albuminuria in the US Third National Health and Nutrition Examination Survey (NHANES III) population: associations with diabetes and level of CKD. Am J Kidney Dis 2006;48(5):720-726

15 Krop JS, Coresh J, Chambless LE, Shahar E, Watson RL, Szklo M et al. A community-based study of explanatory factors for the excess risk for early renal function decline in blacks vs. whites with diabetes: the Atherosclerosis Risk in Communities study. Arch Intern Med 1999; 159(15):1777-1783

16 Tarver-Carr ME, Powe NR, Eberhardt MS, LaVeist TA, Kington RS, Coresh J et al. Excess risk of chronic kidney disease among AfricanAmerican versus white subjects in the United States: a populationbased study of potential explanatory factors. J Am Soc Nephrol 2002; 13(9):2363-2370

17 Hsu CY, Lin F, Vittinghoff E, Shlipak MG. Racial differences in the progression from chronic renal insufficiency to end-stage renal disease in the United States. J Am Soc Nephrol 2003;14(11):2902-2907

18 Peralta CA, Ziv E, Katz R, Reiner A, Burchard EG, Fried L et al. African ancestry, socioeconomic status, and kidney function in elderly African Americans: a genetic admixture analysis. J Am Soc Nephrol 2006; 17(12):3491-3496 
19 Wohland P et al. Life Expectancy variation across England's local areas by ethnic group in 2001. Journal of Maps; 6(1):354-359

20 Ethnicity and ill health. Postnote (276). Parlimetnary Office of Science and Technology. London, 2007

21 Udayaraj et al. Ethnicity, socioeconomic status, and attainment of clinical practice guideline standards in dialysis patients in the United Kingdom. Clin J Am Soc Nephrol 2009;4(5):979-987

22 Navaneethan SD, Singh S. A systematic review of barriers in access to renal transplantation among African Americans in the United States. Clin Transplant 2006;20:769-775

23 Tankersley MR, Gaston RS, Curtis JJ et al. The living donor process in kidney transplantation: influence of race and comorbidity. Transplant Proc 1997;29:3722-3723

24 Lunsford SL, Simpson KS, Chavin KD et al. Racial differences in coping with the need for kidney transplantation and willingness to ask for live organ donation. Am J Kidney Dis 2006;47:324-331

-25 Rudge C et al. Renal transplantation in the United Kingdom for patients from ethnic minorities Transplantation 2007;83(9):1169-1173

26 Loucaidou M et al. Outcome of renal transplantation in south asian recipients is similar to that in non-asians. Transplantation 2004;78: 1021-1024

27 Press R, Carrasquillo O, Nickolas T, Radhakrishnan J, Shea S, Barr RG. Race/ethnicity, poverty status, and renal transplant outcomes. Transplantation 2005;80(7):917-924

28 Isaacs RB, Nock SL, Spencer CE, Connors AF, Jr., Wang XQ, Sawyer R et al. Racial disparities in renal transplant outcomes. Am J Kidney Dis 1999;34(4):706-712

$>29$ Pallet $\mathrm{N}$ et al. Kidney transplant in black recipients: are African Europeans different from African Americans? Am J Transplant 2005; 5(11):2682-2687
30 Schold JD, Kaplan B, Chumbler NR, Howard RJ, Srinivas TR, Ma L et al. Access to quality: evaluation of the allocation of deceased donor kidneys for transplantation. J Am Soc Nephrol 2005;16(10):3121-3127

31 Pei YP, Greenwood CM, Chery AL, Wu GG. Racial differences in survival of patients on dialysis. Kidney Int 2000;58(3):1293-1299

32 Yan $\mathrm{G}$ et al. The relationship of age, race, and ethnicity with survival in dialysis patients. Clin J Am Soc Nephrol 2013;8(6):953-961

33 Roderick, $\mathrm{P}$ et al. Survival of patients from South Asian and Black populations starting renal replacement therapy in England and Wales. Nephrol Dial Transplant 2009 Dec;24(12):3774-3782

34 Lunsford SL, Simpson KS, Chavin KD, Hildebrand LG, Miles LG, Shilling LM et al. Racial differences in coping with the need for kidney transplantation and willingness to ask for live organ donation. Am J Kidney Dis 2006;47(2):324-331

35 Powe NR. Let's get serious about racial and ethnic disparities. J Am Soc Nephrol 2008;19(7):1271-1275

36 Wolfe $\mathrm{M}$ et al. Impact of activated vitamin $\mathrm{D}$ and race on survival among hemodialysis patients. J Am Soc Nephrol 2008 Jul;19(7):1379-1388

37 Nicholas SB et al. Racial disparities in kidney disease outcomes. Semin Nephrol 2013;33(5):409-415

38 Mehrotra R, Kermah D, Fried L, Adler S, Norris K. Racial differences in mortality among those with CKD. J Am Soc Nephrol 2008;19(7): 1403-1410

39 Babayev R et al. Association of race and body mass index with ESRD and mortality in CKD stages 3-4: results from the Kidney Early Evaluation Program (KEEP). Am J Kidney Dis 2013;61(3):404-412 